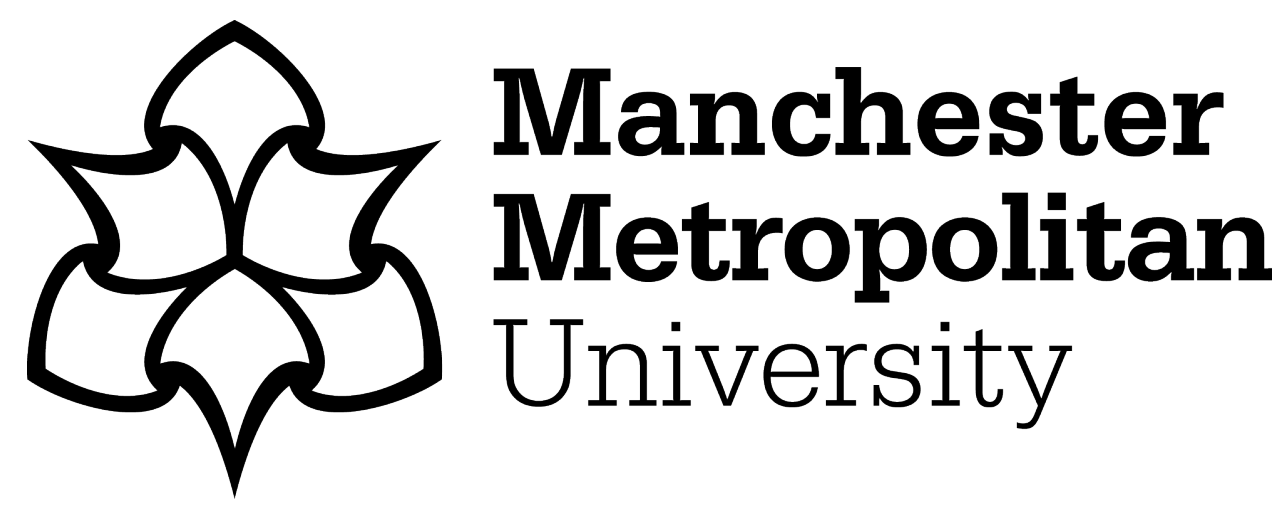

Dos'Santos, T, Comfort, P and Jones, PA (2020) Average of trial peaks versus peak of average profile: impact on change of direction biomechanics. Sports Biomechanics, 19 (4). pp. 483-492. ISSN 1476-3141

Downloaded from: https://e-space.mmu.ac.uk/626018/

Version: Accepted Version

Publisher: Taylor \& Francis

DOI: https://doi.org/10.1080/14763141.2018.1497197

Please cite the published version 


\section{AVERAGE OF TRIAL PEAKS VERSUS PEAK OF AVERAGE PROFILE: IMPACT ON CHANGE OF DIRECTION BIOMECHANICS}

Original Research

Thomas Dos'Santos ${ }^{1}$, Paul Comfort, and Paul A Jones ${ }^{1}$

1 Human Performance Laboratory, Directorate of Sport, Exercise, and Physiotherapy, University of Salford, Greater Manchester, United Kingdom

\section{Correspondence address}

Thomas Dos'Santos

University of Salford

Allerton Building, Frederick Road Campus,

Salford, Greater Manchester, United Kingdom,

M6 6PU.

Telephone: +447961744517

Email: t.dossantos@edu.salford.ac.uk

Abstract word count: 200 words

Manuscript word count: 2929 words

Number of tables and figures: 2 Tables and 2 Figures 


\section{ABSTRACT}

2 The aims of this study were twofold: firstly, to compare lower limb kinematic and kinetic

3 variables during a sprint and $90^{\circ}$ cutting task between two averaging methods of obtaining

4 discrete data (peak of average profile vs average of individual trial peaks); secondly, to

5 determine the effect of averaging methods on participant ranking of each variable within a

6 group. Twenty-two participants, from multiple sports, performed a $90^{\circ}$ cut, whereby lower

$7 \quad$ limb kinematics and kinetics were assessed via 3D motion and ground reaction force (GRF)

8 analysis. Six of the eight dependent variables (vertical and horizontal GRF; hip flexor, knee

9 flexor, and knee abduction moments, and knee abduction angle) were significantly greater ( $p$

$10 \leq 0.001, g=0.10-0.37,2.74-10.40 \%$ ) when expressed as an average of trial peaks compared

11 to peak of average profiles. Trivial $(g \leq 0.04)$ and minimal differences $(\leq 0.94 \%)$ were

12 observed in peak hip and knee flexion angle between averaging methods. Very strong correlations $(\rho \geq 0.901, p<0.001)$ were observed for rankings of participants between averaging methods for all variables. Practitioners and researchers should obtain discrete data based on the average of trial peaks because it is not influenced by misalignments and variations in trial peak locations, in contrast to the peak from average profile.

17 Word count: 2929

Key words: cutting, discrete data, statistical design, kinetics, kinematics 


\section{INTRODUCTION}

Change of directions (COD) are commonly associated with non-contact anterior cruciate ligament injuries in sport (Koga et al., 2010; Olsen, Myklebust, Engebretsen, \& Bahr, 2004). Although the mechanisms of this injury are multifactorial (Quatman, Quatman-Yates, \& Hewett, 2010), lower limb and whole body postures are critical factors associated with knee joint loading (Besier, Lloyd, Cochrane, \& Ackland, 2001; Koga et al., 2010; Kristianslund, Faul, Bahr, Myklebust, \& Krosshaug, 2014; Olsen et al., 2004). Thus, screening athletes' COD biomechanics via the gold standard method of 3D motion analysis (Fox, Bonacci, McLean, Spittle, \& Saunders, 2015) is of great interest to researchers and practitioners to 1) identify the potential mechanisms of injury; 2) identify biomechanical deficits; and 3) risk stratify athletes (Hewett, 2017; Mok \& Leow, 2016).

Lower limb kinetics and kinematic variables including: peak knee abduction angle (KAA), peak knee abduction moment (KAM), peak knee flexion angle, and peak vertical ground reaction force (GRF) are commonly evaluated in athletic populations. These variables have been reported in prospective research to be associated with ACL injury (Hewett et al., 2005; Leppänen et al., 2017) and are also commonly observed characteristics of injury (Hewett, 2017; Koga et al., 2010; Olsen et al., 2004). Potentially 'at-risk' athletes displaying biomechanical deficits in these variables can be subsequently treated and rehabilitated to reduce the relative risk of injury (Hewett, 2017; Mok \& Leow, 2016).

Most COD biomechanical investigations include more than one trial to evaluate biomechanical parameters (Dai et al., 2014; Dempsey, Lloyd, Elliott, Steele, \& Munro, 2009; Havens \& Sigward, 2015; Sigward, Cesar, \& Havens, 2015). Although practitioners may examine COD biomechanics during the fastest trial or examine peak data, which potentially represents the likely demands placed upon an athlete in the 'worst case scenario', evaluating 
47 a single trial does not represent an athlete's typical and generalised movement (James, Herman, Dufek, \& Bates, 2007). Moreover, single trial protocols have been suggested to be invalid and unreliable which could lead to erroneous conclusions (Bates, Dufek, \& Davis, 1992; James et al., 2007). As such, practitioners and researchers average biomechanical parameters across multiple trials to represent an athlete's generalised and typical movement. However, one issue in evaluating kinematic and kinetics via 3D motion analysis is the method of analysing and obtaining discrete data from specific events (i.e. maximum or minimum values during weight acceptance), and whether to obtain discrete data from the peak of an average curve/profile or to average the individual trial peaks. Recently, Dames, Smith, and Heise (2017) demonstrated angular velocity $(p<0.001, E S=0.08-0.16,1.1-2.2 \%)$ and initial vertical GRF ( $p=0.002, E S=0.09,0.9 \%$ ) peak values from the average profile were significantly lower compared to averaging trial peaks during walking gait $(1.5 \mathrm{~m} / \mathrm{s})$. The authors subsequently recommended parameters should be obtained from averaging trial peaks compared to peak of average profile for a better representation of the data. To our knowledge, no averaging recommendations exist for obtaining COD parameters.

Unfortunately, it remains unclear in the COD biomechanical literature how researchers derive discrete data as several studies state trials were averaged (Besier et al., 2001; Havens \& Sigward, 2015; Sigward et al., 2015), but do not delineate whether a peak of an average profile or average of trials peaks method was used. Additionally, some studies fail to state whether average data was used for statistical analysis (Dai et al., 2016; Dai et al., 2014; Dempsey et al., 2009). This is concerning because failing to state averaging procedures makes it difficult to facilitate methodological replication, and if different outcome values are produced between averaging methods, this could lead to different evaluations and diagnoses in clinical and laboratory environments. 
The aims of this study were twofold: firstly, to compare lower limb kinematic and

kinetic variables during a sprint and $90^{\circ}$ cutting task between two averaging methods of obtaining discrete data (peak of average profile vs average of individual trial peaks); secondly, to determine the effect of averaging methods on participant ranking of each variable within a group. It was hypothesised that different values would be produced between the two averaging methods; however, there would be minimal differences in the ranking of athletes.

\section{METHODS}

\section{Participants}

Twenty-two (15 male and 7 female age: $23.2 \pm 4.4$ years, mass: $74.9 \pm 12.8 \mathrm{~kg}$, height: $1.75 \pm$ $0.09 \mathrm{~m}$ ) athletes from multiple sports (soccer $n=11$, rugby $n=4$, netball $n=5$, cricket $n=2$ ) participated in this study, which was greater than the sample size $(n=12)$ used by Dames et al. (2017) who examined the effect of averaging method on walking gait outcomes. The investigation was approved by the University of Salford institutional ethics review board, and all participants provided written informed consent. All participants performed a 5-minute warm up consisting of jogging, self-selected dynamic stretching, and familiarisation trials of the $90^{\circ}$ cuts ( 4 trials performed submaximally at $75 \%$ of perceived maximal effort); similar to the warm up procedures utilised in previous studies (Dai et al., 2014; Vanrenterghem, Venables, Pataky, \& Robinson, 2012).

\section{Experimental protocol}

Lower limb kinematic and kinetic data were collected during a $90^{\circ}$ cut to the left $(5 \mathrm{~m}$ entry and $3 \mathrm{~m}$ exit - push off right leg), performed as fast as possible (completion time $2.07 \pm 0.09$ seconds), on an indoor track (Mondo, SportsFlex, $10 \mathrm{~mm}$; Mondo America Inc., Mondo, 
Summit, NJ, USA). Participants performed a minimum of three acceptable trials (Mok, Bahr, \& Krosshaug, 2017). If the participants slid, turned prematurely, or missed the force platform, the trial was discarded and subsequently another trial was performed after two minutes rest.

The procedures were based on previously published protocols (Jones, Herrington, \& Graham-Smith, 2016; Jones, Thomas, Dos'Santos, McMahon, \& Graham-Smith, 2017), thus a brief overview is provided. Prior to the cutting task, reflective markers (14 $\mathrm{mm}$ spheres) were placed on bony landmarks of each participant (Jones et al., 2016; Jones et al., 2017). Each participant wore a 4-marker "cluster set" (4 retroreflective markers attached to a lightweight rigid plastic shell) on the right and left thigh and shin which approximated the motion of these segments during the dynamic trials. All participants wore lycra shorts and female participants wore a compression top (Champion Vapor, Champion, Winston-Salem, NC, USA). Standardised footwear (Balance W490, New Balance, Boston, MA, USA) was provided for all participants to control for shoe-surface interface.

\section{Data analysis}

Three dimensional motions of these markers were collected during the cutting trials using 10 Qualisys Oqus 7 (Gothenburg, Sweden) infrared cameras $(240 \mathrm{~Hz}$ ) operating through Qualisys Track Manager software (Qualisys, version 2.16, build 3520, Gothenburg, Sweden). The GRF's were collected from one $600 \mathrm{~mm} \times 900 \mathrm{~mm}$ AMTI (Advanced Mechanical Technology, Inc, Watertown, MA, USA) force platform (Model number: 600900) embedded into the running track sampling at $1200 \mathrm{~Hz}$. From a standing trial, a lower extremity and trunk 6 degrees of freedom kinematic model was created for each participant, including pelvis, thigh, shank, and foot using Visual 3D software (C-motion, version 6.01.12, Germantown, USA). This kinematic model was used to quantify the motion at the hip, knee, and ankle joints using a Cardan angle sequence x-y-z (Suntay, 1983). The local coordinate system was defined at the proximal joint centre for each segment. The static trial position was 
designated as the participant's neutral (anatomical zero) alignment, and subsequent kinematic and kinetic measures were related back to this position. Segmental inertial characteristics were estimated for each participant (Dempster, 1955). This model utilised a CODA pelvis orientation (Bell, Brand, \& Pedersen, 1989) to define the location of the hip joint centre. The knee and ankle joint centres were defined as the mid-point of the line between lateral and medial markers. Lower limb joint moments were calculated using an inverse dynamics approach (Winter, 2009) through Visual 3D software and were defined as external moments.

Initial contact was defined as the instant after ground contact that the vertical GRF was higher than $20 \mathrm{~N}$, and end of contact was defined as the point where the vertical GRF subsided past $20 \mathrm{~N}$ (Kristianslund et al., 2014). The weight acceptance phase was defined as the instant of initial contact to the point of maximum knee flexion (Havens \& Sigward, 2015). All joint moments, joint angles, and GRFs (see table 1 for dependent variables) were derived during weight acceptance and used for further analysis. Using the pipeline function in visual 3D, joint coordinate and force data were smoothed with a fourth-order Butterworth low-pass digital filter with cut-off frequencies of $15 \mathrm{~Hz}$ and $25 \mathrm{~Hz}$, based on a priori residual analysis (Winter, 2009), visual inspection of motion data, and recommendations by Roewer, Ford, Myer, and Hewett (2014).

An average magnitude of each dependent variable were derived using two methods (Dames et al., 2017) and compared for statistical analysis. The first involved averaging the individual trial peaks across three trials (average of trial peaks). The second involved creating an average profile (normalised to $100 \%$ of weight acceptance) of three trials and obtaining a single peak from the average profile (peak from average profile).

\section{Statistical Analyses}

All statistical analysis was performed in SPSS v 23 (SPSS Inc., Chicago, IL, USA) and Microsoft Excel (version 2016, Microsoft Corp., Redmond, WA, USA). Normality was 
confirmed for all variables utilising a Shapiro-Wilks test. Within-session reliability was assessed using intra class coefficients (ICC) and the standard error of measurement was calculated using the formula: $S D($ pooled $) * \sqrt{(1-\text { ICC })}$ (Thomas, 2005). Differences in dependent variables between methods were assessed using paired sample t-tests, effect sizes, mean differences (bias), and percentage differences. The 95\% limits of agreement (LOA) (LOA: mean of the difference \pm 1.96 standard deviations) were also calculated between averaging methods using methods described by Bland and Altman (1986). All data was visually inspected using Bland-Altman plots to confirm homoscedasticity. Hedges' $g$ effect sizes were calculated as described previously (Hedges \& Olkin, 1985) and interpreted using Hopkins' (2002) scale. To assess whether averaging method impacted the ranking of players for each dependent variable, Spearman's correlations coefficients were also calculated. Statistical significance was defined $p \leq 0.05$ for all tests.

\section{RESULTS}

Mean \pm SD are presented for all dependent variables between averaging methods in Table 1 . All variables demonstrated high within-session reliability measures $($ ICC $\geq 0.863)$ and SEM values ranged from $0.08-0.10 \mathrm{~N} / \mathrm{BW}$ for GRF variables, $1.10-2.64^{\circ}$ for joint angles, and $0.12-$ $0.23 \mathrm{Nm} / \mathrm{kg}$ for joint moments (Table 1).

Six of the 8 dependent variables (vertical and horizontal GRF, hip flexor, knee flexor, and knee abduction moments, and knee abduction angle) were significantly greater when expressed as an average of trial peaks compared to peak of average profiles, with trivial to small effect sizes $(g=0.10-0.37)$ and mean percentage differences of $2.74-10.40 \%$ (Table 1$)$, respectively. Although a statistical, significant difference was observed in peak hip flexion angle between the two averaging methods, effect sizes $(g=0.04)$ and percentage differences $(0.9 \%)$ indicated a trivial and minimal difference (Table 1). Similarly, a non-significant, 
trivial, and minimal percentage difference $(p=0.279, g=0.01,0.09 \%)$ was observed in peak knee flexion angle between averaging methods (Table 1). Very strong correlations ( $\rho \geq 0.901$, $p<0.001)$ were observed for rankings of participants between averaging methods for all dependent variables (Table 2).

***Insert Table 1 about here***

***Insert Table 2 about here***

\section{DISCUSSION AND IMPLICATIONS}

The aims of this study were to examine the impact of averaging methods (peak of average profile vs average of individual trial peaks) on commonly examined lower limb kinematic and kinetic variables during cutting, and to determine the effect of averaging method on participant ranking. The primary findings were significantly lower GRF, joint moments, and KAA values were demonstrated when obtaining peak of average profile data compared to average of trial peaks data (Table 1), supporting the study hypothesis. These results are in line with the findings of Dames et al. (2017) that reported significantly lower angular velocity $(p<0.001, E S=0.08-0.16,1.1-2.2 \%)$ and GRF $(p=0.002, E S=0.09,0.9 \%)$ data during walking gait $(1.5 \mathrm{~m} / \mathrm{s})$, based on peak of average profile data. Interestingly, the averaging method had a trivial and minimal effect on sagittal plane joint angles (hip and knee flexion) (Table 1). Additionally, very strong correlations were observed for participant ranking between averaging methods for all dependent variables (Table 2), indicating an athlete will most likely achieve the same ranking in a cohort of athletes, irrespective of the averaging method used. 
The trivial to small, yet significantly lower values $(2.74-10.40 \%)$ observed in the present study for GRF, joint moment, and KAA (Table 1) variables could be attributed to misalignments in the temporal profiles (variation in the location of peak data on the time series) (Figure 1) (Dames et al., 2017). It is worth noting, however, that if trial peaks occur at a similar point of the time-series (i.e. similar $\%$ of weight acceptance), then the differences between averaging methods will be minimal, evident by the minimal differences observed for peak hip and knee flexion angles in the present study (Table 1). Nevertheless, when the individual trials are normalised to produce an average profile, the peak value from these curves were on average $2.74-10.40 \%$ lower for GRF, joint moment, and KAA variables. The subtle differences in values may lead to different evaluations and diagnoses in clinical and laboratory environments; thus, researchers and practitioners are consequently recommended to standardise the averaging method when longitudinally monitoring changes in COD biomechanical parameters to allow valid comparisons. Furthermore, researchers are recommended to clearly state their averaging method to facilitate methodological replication. Future applied work could consider determining a phase shift to remove large outliers in the data set (Dames et al., 2017).

$$
\text { ***Insert Figure } 2 \text { about here*** }
$$

In contrast to Dames et al. (2017), that found greater magnitudes of differences for kinematic (angular velocities) data than kinetic (GRF) data between averaging methods, the present study found the largest effect sizes between averaging methods were present for GRF data (Table 1, Figure 2). These opposing findings could be explained by Dames et al. (2017) investigating walking gait which is lower in velocity compared to the present study and thus, associated with lower GRF's, particularly horizontally. Additionally, cutting is a more complex manoeuvre than walking, whereby the addition of higher entry velocity most likely results in slightly different movements strategies at impact between trials, thus resulting in 
temporal misalignments in peak GRF (Figure 2). Finally, it should be acknowledged that joint angular velocities were the only kinematic variables examined by Dames et al. (2017), whereas the present study examined peak joint angles over weight acceptance during cutting, whereby sagittal plane joint angles hip and knee flexion demonstrated consistent temporal alignments, thus minimal differences.

Caution is advised when using pre-defined thresholds in order to identify potentially 'at-risk' athletes for particular parameters which may be used to subsequently inform training. For example, an athlete below an 'at-risk' threshold may not be classified as 'at risk' for a particular variable based on peak of average profile data. Conversely, based on average of trial peaks data the same athlete may have a greater value which subsequently classes them as 'at-risk', and may therefore receive specific training or treatment. It could therefore, be argued that the choice of averaging method could lead to different clinical diagnoses, and may result in false negative/positive identification which could influence the future training for that athlete. However, it is worth noting that very strong relationships were observed for participant ranking between averaging methods for all dependent variables (Table 2), indicating an athlete will most likely achieve the same ranking in a cohort of athletes, irrespective of the averaging method used.

It is also worth acknowledging that standard deviations (variation) observed for kinetic and kinetic variables (Table 1) were similar between averaging methods, in line with the findings of Dames et al. (2017). Thus, it is likely that absolute reliability may be similar between averaging methods based on standard deviation driven reliability measures. Further research comparing the effect of these aforementioned averaging methods on betweensession reliability is warranted to confirm which method provides the best reliability and most sensitive measures. 
It should be noted that the present study has several limitations. Firstly, by averaging

242 trials, in essence, a mythical, never performed trial is created; however, this procedure is commonly used within the COD literature (Besier et al., 2001; Havens \& Sigward, 2015; Jones et al., 2016; Jones et al., 2017; Sigward et al., 2015), and averaging trials is suggested to be representative of a participant's typical movement (James et al., 2007). Secondly, while it may be common practice to average parameters between trials, some researchers may choose to evaluate the fastest trial (Kimura \& Sakurai, 2013), or the trial displaying the greatest biomechanical deficit (i.e. greatest KAM or KAA) may also be of interest, though caution is advised when evaluating movement based on a single trial (Bates et al., 1992; James et al., 2007).

\section{CONCLUSION}

In conclusion, the averaging method to obtain discrete data results in subtle differences in values produced, with the peak from the average profile demonstrating lower GRF, joint moment, and KAA values during cutting. Consequently, researchers and practitioners are recommended to obtain discrete data based on an average of trial peaks because it is not influenced by misalignments and variations in trial peak locations, in contrast to the peak from average profile. However, with the respect to participant ranking, minimal differences are present between averaging methods. Researchers and practitioners are also recommended to standardise the averaging method when longitudinally monitoring changes in COD biomechanics for screening and clinical purposes or making group comparisons. Moreover, when publishing research, it is advocated that researchers clearly state the averaging method implemented to facilitate methodological replication. 


\section{DISCLOSURE STATEMENT}

No potential conflict of interest was reported by the authors

\section{ACKNOWLEDGEMENTS}

The authors confirm would like to thank the participants for participating in this study.

\section{REFERENCES}

Bates, B. T., Dufek, J. S., \& Davis, H. P. (1992). The effect of trial size on statistical power. Medicine and science in sports and exercise, 24(9), 1059-1065.

Bell, A. L., Brand, R. A., \& Pedersen, D. R. (1989). Prediction of hip joint centre location from external landmarks. Human Movement Science, 8(1), 3-16.

Besier, T. F., Lloyd, D. G., Cochrane, J. L., \& Ackland, T. R. (2001). External loading of the knee joint during running and cutting maneuvers. Medicine \& Science in Sports \& Exercise, 33(7), 11681175.

Bland, J. M., \& Altman, D. (1986). Statistical methods for assessing agreement between two methods of clinical measurement. The lancet, 327(8476), 307-310.

Dai, B., Garrett, W. E., Gross, M. T., Padua, D. A., Queen, R. M., \& Yu, B. (2016). The effect of performance demands on lower extremity biomechanics during landing and cutting tasks. Journal of Sport and Health Science, 43(9), 1680-1608.

Dai, B., William, E. G., Michael, T. G., Darin, A. P., Robin, M. Q., \& Bing, Y. (2014). The Effects of 2 Landing Techniques on Knee Kinematics, Kinetics, and Performance During Stop-Jump and Side-Cutting Tasks. The American journal of sports medicine, 43(2), 466-474. doi:doi:10.1177/0363546514555322

Dames, K. D., Smith, J. D., \& Heise, G. D. (2017). Averaging Trials Versus Averaging Trial Peaks: Impact on Study Outcomes. J Appl Biomech, 33(3), 233-236. doi:10.1123/jab.2016-0164

Dempsey, A. R., Lloyd, D. G., Elliott, B. C., Steele, J. R., \& Munro, B. J. (2009). Changing sidestep cutting technique reduces knee valgus loading. The American journal of sports medicine, 37(11), 2194-2200.

Dempster, W. T. (1955). Space requirements of the seated operator: geometrical, kinematic, and mechanical aspects of the body, with special reference to the limbs.

Fox, A. S., Bonacci, J., McLean, S. G., Spittle, M., \& Saunders, N. (2015). A Systematic Evaluation of Field-Based Screening Methods for the Assessment of Anterior Cruciate Ligament (ACL) Injury Risk. Sports Medicine, 1-21.

Havens, K. L., \& Sigward, S. M. (2015). Cutting mechanics: relation to performance and anterior cruciate ligament injury risk. Medicine and science in sports and exercise, 47(4), 818-824.

Hedges, L., \& Olkin, I. (1985). Statistical Methods for Meta-Analysis. New York: Acdemic Press.

Hewett. T. (2017). Preventive biomechanics: A paradigm shift with a translational approach to biomechanics. Journal of Science and Medicine in Sport, 20, e67.

Hewett, T., Myer, G. D., Ford, K. R., Heidt, R. S., Colosimo, A. J., McLean, S. G., . . Succop, P. (2005). Biomechanical measures of neuromuscular control and valgus loading of the knee predict anterior cruciate ligament injury risk in female athletes a prospective study. The American journal of sports medicine, 33(4), 492-501.

Hopkins, W. G. (2002). A scale of magnitudes for effect statistics. A new view of statistics. 
James, C. R., Herman, J. A., Dufek, J. S., \& Bates, B. T. (2007). Number of trials necessary to achieve performance stability of selected ground reaction force variables during landing. Journal of sports science \& medicine, 6(1), 126.

Jones, P., Herrington, L., \& Graham-Smith, P. (2016). Braking characteristics during cutting and pivoting in female soccer players. Journal of Electromyography and Kinesiology, 30, 46-54.

Jones, P., Thomas, C., Dos'Santos, T., McMahon, J., \& Graham-Smith, P. (2017). The Role of Eccentric Strength in $180^{\circ}$ Turns in Female Soccer Players. Sports, 5(2), 42.

Kimura, K., \& Sakurai, S. (2013). A Sidestep Cut Preparation Strategy Decreases the External Load Applied to the Knee Joint. International Journal of Sport and Health Science, 11, 109-117.

Koga, H., Nakamae, A., Shima, Y., Iwasa, J., Myklebust, G., Engebretsen, L., . . Krosshaug, T. (2010). Mechanisms for noncontact anterior cruciate ligament injuries knee joint kinematics in 10 injury situations from female team handball and basketball. The American journal of sports medicine, 38(11), 2218-2225.

Kristianslund, E., Faul, O., Bahr, R., Myklebust, G., \& Krosshaug, T. (2014). Sidestep cutting technique and knee abduction loading: implications for ACL prevention exercises. British Journal of Sports Medicine, 48(9), 779-783.

Leppänen, M., Pasanen, K., Kujala, U. M., Vasankari, T., Kannus, P., Äyrämö, S., . . Perttunen, J. (2017). Stiff landings are associated with increased ACL injury risk in young female basketball and floorball players. The American journal of sports medicine, 45(2), 386-393.

Mok, K.-M., Bahr, R., \& Krosshaug, T. (2017). Reliability of lower limb biomechanics in two sportspecific sidestep cutting tasks. Sports Biomechanics, 1-11.

Mok, K.-M., \& Leow, R.-S. (2016). Measurement of movement patterns to enhance ACL injury prevention"A dead end? Asia-Pacific Journal of Sports Medicine, Arthroscopy, Rehabilitation and Technology, 5, 13-16.

Olsen, O.-E., Myklebust, G., Engebretsen, L., \& Bahr, R. (2004). Injury mechanisms for anterior cruciate ligament injuries in team handball a systematic video analysis. The American journal of sports medicine, 32(4), 1002-1012.

Quatman, C. E., Quatman-Yates, C. C., \& Hewett, T. E. (2010). A 'Plane'Explanation of Anterior Cruciate Ligament Injury Mechanisms. Sports Medicine, 40(9), 729-746.

Roewer, B. D., Ford, K. R., Myer, G. D., \& Hewett, T. E. (2014). The 'impact' of force filtering cut-off frequency on the peak knee abduction moment during landing: artefact or 'artifiction'? $\mathrm{Br} J$ Sports Med, 48(6), 464-468. doi:10.1136/bjsports-2012-091398

Sigward, S. M., Cesar, G. M., \& Havens, K. L. (2015). Predictors of frontal plane knee moments during side-step cutting to 45 and 110 degrees in men and women: implications for anterior cruciate ligament injury. Clinical journal of sport medicine, 25(6), 529-534.

Suntay, W. (1983). A joint coordinate system for the clinical description of three-dimensional motions: application to the knee. Journal of biomechanical engineering, 105, 136-144.

Thomas, J. R., Nelson, JK., Silverman SJ. (2005). Research Methods in Physical Activity. Champaign, IL: Human Kinetics.

Vanrenterghem, J., Venables, E., Pataky, T., \& Robinson, M. A. (2012). The effect of running speed on knee mechanical loading in females during side cutting. Journal of biomechanics, 45(14), 2444-2449.

Winter, D. A. (2009). Biomechanics and motor control of human movement: John Wiley \& Sons. 
Table 1. Comparisons in dependent variables between averaging methods

\begin{tabular}{|c|c|c|c|c|c|c|c|c|c|c|c|c|c|c|}
\hline \multirow{2}{*}{ Variable } & \multicolumn{2}{|c|}{$\begin{array}{c}\text { Average of trial } \\
\text { peaks }\end{array}$} & \multicolumn{2}{|c|}{$\begin{array}{c}\text { Peak of average } \\
\text { profile }\end{array}$} & \multirow[t]{2}{*}{$p$} & \multirow[t]{2}{*}{$g$} & \multicolumn{2}{|c|}{ Mean difference (Bias) } & \multicolumn{2}{|c|}{$95 \%$ LOA } & \multicolumn{2}{|c|}{$\%$ difference } & \multirow{2}{*}{ ICC } & \multirow{2}{*}{$\begin{array}{l}3611 \\
362\end{array}$} \\
\hline & Mean & SD & Mean & SD & & & Mean & SD & LB & UB & Mean & SD & & \\
\hline pk VGRF (N/BW) & 2.55 & 0.44 & 2.39 & 0.40 & $<0.001$ & 0.37 & 0.15 & 0.13 & -0.11 & 0.42 & 5.93 & 5.12 & 0957 & $\begin{array}{l}0.10 \\
363\end{array}$ \\
\hline pk HGRF (N/BW) & -1.41 & 0.27 & -1.33 & 0.25 & $<0.001$ & -0.30 & -0.11 & 0.02 & -0.21 & 0.05 & 5.58 & 4.08 & 0.933 & 3064 \\
\hline pk HFA $\left(^{\circ}\right)$ & 47.52 & 10.0 & 47.07 & 9.95 & 0.001 & 0.04 & 0.45 & 0.54 & -0.6 & 1.51 & 0.94 & 1.10 & 0.962 & $\begin{array}{l}365 \\
2.02 \\
366\end{array}$ \\
\hline pk HFM (Nm/kg) & -2.65 & 0.91 & -2.46 & 0.90 & 0.001 & 0.21 & 0.19 & 0.23 & -0.63 & 0.25 & 7.31 & 8.37 & 0.941 & 3628 \\
\hline pk KFA $\left(^{\circ}\right)$ & 59.77 & 6.44 & 59.73 & 6.50 & 0.279 & 0.01 & 0.04 & 0.19 & -0.33 & 0.42 & 0.09 & 0.38 & 0.863 & $\begin{array}{l}368 \\
2.64 \\
369\end{array}$ \\
\hline pk KFM (Nm/kg) & 3.46 & 0.63 & 3.37 & 0.63 & $<0.001$ & 0.15 & 0.09 & 0.09 & -0.09 & 0.28 & 2.74 & 2.69 & 0.936 & 3070 \\
\hline pk KAA $\left(^{\circ}\right)$ & -8.16 & 8.09 & -7.31 & 8.09 & $<0.001$ & -0.10 & -0.86 & 0.92 & -2.66 & 0.95 & 10.40 & 9.41 & 0.982 & $\begin{array}{l}371 \\
1.10 \\
372\end{array}$ \\
\hline pk KAM (Nm/kg) & 0.99 & 0.36 & 0.92 & 0.34 & $<0.001$ & 0.20 & 0.07 & 0.07 & -0.07 & 0.21 & 7.03 & 6.74 & 0.909 & 30713 \\
\hline
\end{tabular}

Key: pk: Peak; VGRF: Vertical ground reaction force; HGRF: Horizontal ground reaction force; HFA: Hip flexion angle; HFM: Hip flexor moment; KFA: Knee flexion angle; KFM: Knee 374 flexor moment; KAA: Knee abduction angle; KAM: Knee abduction moment; LOA: Limits of agreement; LB: Lower bound; UB: Upper bound; BW: Body weight; ICC: Intraclass correlaß̊7̧ coefficient; SEM: Standard error of measurement
377
378
379
380
381
382
383
384
385 
Table 2. Spearman's correlations based on ranking of participants between averaging methods for dependent variables

\begin{tabular}{ccc}
\hline Dependent variable & $\rho$ & p value \\
\hline pk VGRF & 0.901 & $<0.001$ \\
pk HGRF & 0.961 & $<0.001$ \\
pk HFA & 0.997 & $<0.001$ \\
pk HFM & 0.958 & $<0.001$ \\
pk KFA & 1.000 & $<0.001$ \\
pk KFM & 0.960 & $<0.001$ \\
pk KAA & 0.983 & $<0.001$ \\
pk KAM & 0.964 & $<0.001$
\end{tabular}

Key: pk: Peak; VGRF: Vertical ground reaction force; HGRF:

Horizontal ground reaction force; HFA: Hip flexion angle; HFM: Hip

flexor moment; KFA: Knee flexion angle; KFM: Knee flexor moment;

KAA: Knee abduction angle; KAM: Knee abduction moment 